How To ...

\title{
Description of Nasoalveolar Molding Process in Neonatal Period of Unilateral Cleft Lip and Palate: A Step by Step
}

\author{
Mohamed Azhari, Wijdane El Hawari (D), Hasnae Rokhssi, Nadia Merzouk, Oussama Bentahar \\ Department of Prosthodontics, Faculty of Dental Medicine, University Mohammed V, Rabat, Morocco
}

\begin{abstract}
Statement of Problem. Cleft Lip and Palate (CLP) are common congenital defects of the present day. They result from a failure of facial bud sticking during embryological phenomena. Their therapeutics based on multi-disciplinary care to restore as much as possible the aesthetic and functional prejudices generated by these anomalies. Purpose: Through this work, we will illustrate, through a clinical case supported at the Consultation and Dental Treatment Center of Rabat, clinical and laboratory stages of realization of Nasoalveolar Molding (NAM). Material and Methods: We describe original techniques used in our service for manufacturing of NAM fireworks and we define recommendations after insertion and follow-up procedures.Results: Place of NAM in management of these anomalies has increasingly recommended, given limitations of surgery alone to ensure satisfactory aesthetic results. Several fixed or removable appliances has used, in order to reposition gaps in cleft and / or to reshape affected bordering tissues. Conclusion: Despite the controversies surrounding this therapy, it remains highly recommended and scientifically based.
\end{abstract}

KEYWORDS: Cleft Lip and Palate; Nasoalveolar: Molding.

Correspondence: Dr. El Hawari Wijdane. Email: wijdane.elhawari@um5s.net.ma \& Dr. Mohamed Azhari. Email: mohamed.azhari@um5s.net.ma. Department of Prosthodontics, Faculty of Dental Medicine, University Mohammed V, Rabat. Avenue Allal Al Fassi, Rue Mohammed Jazoulit, Madinat Al Irfane, BP: 6212, Rabat, Morocco.

Copyright $\odot 2021$ Azhari $M$ et al. This is an open access article distributed under the Creative Commons Attribution 4.0 International, which permits unrestricted use, distribution, and reproduction in any medium, provided the original work is properly cited.

\section{INTRODUCTION}

Nasoalveolar Molding process in the global protocol for the management of CLP has approved for several years. It is started early in the neonatal phase, and continues after labio-alveolar plastic surgery, usually started at 6 months after birth. This orthopedic therapy parallel to surgical therapy ensures a recovery of cartilage constituting the collapsed nose wing, the columella often inclined towards the split side and contributes to a rapprochement of the lips, which facilitates surgical act. The NAM does not only targets the labio-nasal soft tissues, but also bone support tissues. Terminologically, term "molding" has used to describe the action on soft tissues and "orthopedic" action on bone tissues. Nevertheless, it should not be denied that soft tissue molding is also an "indirect orthopedic" because muscular and cartilaginous changes brought about by influence modeling of the underlying support bone. Thus, by ensuring the approximation of the lips, it influences a centripetal action on the two fragments of the jaws bordering cleft.

Thus, we must not neglect role played by palatal plate, which in addition to its obturation of oral communication often associated with CLP, it allows performing a direct orthopedic action, via an action on the edges of cleft, by adding acrylic resin at its lower surface. This for facing the small fragment to expel it to the outside, and allow having a continuity of the alveolar arch. In addition, it allows indirect orthopedics by favoring a low position of the tongue, preventing it from coming to be introduce at the level of the cleft, and thus avoids accentuating the inter-fragment space.

Through this work, we will describe, through one clinical case supported at the Consultation and Dental Treatment Center of Rabat (CDTCR), the equipment used in the context of NAM, as well as the clinical stages of their elaboration, by relating original techniques of our service, then the follow-up protocol.

\section{CLINICAL REPORT}

Case History, Examination, and Diagnosis

This a 2-day-old infant suffering from complete unilateral cleft lip and palate (right side) presented to the Department of Prosthodontics at Dental Faculty and Hospital of Rabat, for evaluation and treatment. A 
general physical examination was carried out under the supervision of the physician, and consent was obtained to start the molding procedure in the first week of birth. A cleft defect was examined for the presence of natal teeth, unusual undercuts, and other tissue abnormalities. The distance between the two alveolar segments was $11 \mathrm{~mm}$ (Fig. 1A)

Impression, Working Cast, and Fabrication of Oral Molding Appliance

Following evaluation and a thorough explanation of the treatment goals and the procedure to the parents, an impression of an intraoral cleft defect was made using an Artisanal Impression Tray adjusted on a newborn model previously supported (Fig.1B). With the infant fully awake and without any anesthesia in a clinical setting prepared to handle an airway emergency with a surgeon present as a part of the impression team, the impression was obtained. The infant was held in an upside down position by the surgeon, and the impression tray was inserted into the oral cavity. The tray was seated until the impression material was observed just beginning to extrude past its posterior border. After application of the universal adhesive, the impression has made using a silicone OPTOSIL ${ }^{\circledR}$, with a slight digital pressure starting first by the posterior sectors, then by tilting towards the anterior sector, to get more material escaping to the labial area (Fig.1C). This impression has rebased, for better recording accuracy, using a fluid elastomer XANTOPREN® (Fig.1D). During impression making, the infant was held in an inverted position to keep the tongue forward and to allow fluids to drain out of the oral cavity. Once the impression material was set, the tray was removed, and the oral cavity examined for the residual impression material in the cleft region. An impression was carefully poured in type III gypsum product, and the cast was recovered. The size of the cleft defect was measured at the base of the alveolus on the cast using a vernier caliper and was found to be $11 \mathrm{~mm}$. The cleft region of the palate and alveolus filled in with baseplate wax to approximate the contour and topography of an intact arch before the fabrication of the oral portion of the molding appliance (Fig. 1E). The cast was duplicated in irreversible hydrocolloid to obtain a working cast on which two layers of baseplate wax were adapted and lab processed using clear heat-cure acrylic resin to fabricate the molding prosthesis of 2 to $3 \mathrm{~mm}$ thickness to provide structural integrity and to permit adjustments during the molding therapy. The appliance was finished and polished to ensure that all tissue borders were smooth and that the oral portion of the appliance that would be in contact with the dorsum of the tongue given a high polish (Fig. 1F). At the insertion appointment, the appliance has carefully fitted in the infant's oral cavity and observed for few minutes. The infant was able to suckle without gagging or struggling. The patient was recalled on a weekly basis for follow-up. The acrylic has selectively removed from the inner labial aspect of the lesser segment of the alveolus $(1 \mathrm{~mm})$ while adding an equal amount of permanent soft liner on the palatal aspect to direct the lesser segment outward from the cleft (Fig. 2A).

\section{Nasal Stent}

The phase of active nasal cartilage molding began when the intraalveolar gap reduced to approximately $5 \mathrm{~mm}$ by incorporation of the nasal stent component. The rationale behind delaying the addition of nasal stent was that with a reduced alveolar gap, the base of the nose and lip segment alignment was improved. At this stage, a second impression was made, and the procedure was repeated. A nasal stent constructed from 0.018 inch round stainless steel wire was secured to the labial flange of the appliance. The wire extending into the nostril was curved back on itself to create a small loop for retention of the intranasal hard acrylic component of the nasal stent to provide form and support to the tissues. This hard acrylic component was shaped into a bilobed form resembling a kidney. Periodic examination of the tissues and adjustment of the appliance was continued every week to mold the nasoalveolar complex into the desired shape and position. After 4 months, the intraalveolar gap between the two segments at the crestal level was approximately 2 $\mathrm{mm}$, ensuring a clinically desirable approximation of the alveolar segments. After completion of the PNAM procedure, the alveolar segments were aligned, and the nasal cartilages, columella, and philtrum were properly repositioned (Fig. 2B). The infant has scheduled for surgical repair with the plastic surgeon after parents' consent (Fig. 2C).

\section{Surgical Procedure}

The primary surgical closure of the lip and nose has performed at 4 months of age (Fig. 2D). The surgical technique modified to take the advantage of the Presurgical NAM preparation. Because the alveolar segments were in approximation, a gingivoperiosteoplasty (GPP) made it simple for the plastic surgeon to perform palatal and alveolar closure. The patient was followed regularly at 3-month intervals.

\section{Post-Surgical Nostril Retainer}

After closure of the upper lip, the wire has removed and the palatal plate has adjusted to the level of the new anterior vestibule floor, through a silicone impression after anterior fenestration followed by relining in the laboratory. The nostril retainer replace the nasal button. For this, an extra oral impression was made using a steel wire was shaped in the form of a fork, with two ends and a handle. First, it was verified that the ends penetrate without interference at the nasal level. The thread has buried in a quantity of silicone OPTOSIL $\AA$, and the whole has introduced into the nostrils. After complete capture of the impression material, its handle left outside removed the fork. Then, the impression has rebased with a low viscosity elastomer XANTOPREN® (Fig. 2 E, F). After casting the impression, we obtain a nasal model (Fig 3. A). Then, two $1.5 \mathrm{~mm}$ diameter metal rods has fixed at the nasal level with two drops of sticky wax. Then, the model was isolated with a laboratory varnish ISOPLAST®. Subsequently, the transparent selfpolymerizing resin ORTHORESIN® was casted at the level of the nostrils until the filling of their volume, and connected, a posteriori, by a columellar bridge of a thickness not exceeding $1 \mathrm{~mm}$. After complete polymerization, the molding was fractionated and the nostril retainer was removed. Successively, the two stems 
has removed, leaving in place two orifices that will allow releasing the nasal breathing (Fig. 3B). After roughing, finishing and polishing, the nostril retainer has inserted. Control sessions were scheduled each month. The nostril retainer was subsequently adapted and held in place with a band of plaster (Fig 3C, D). Follow-up has performed monthly by adding the resin to the valve at the split nostril.

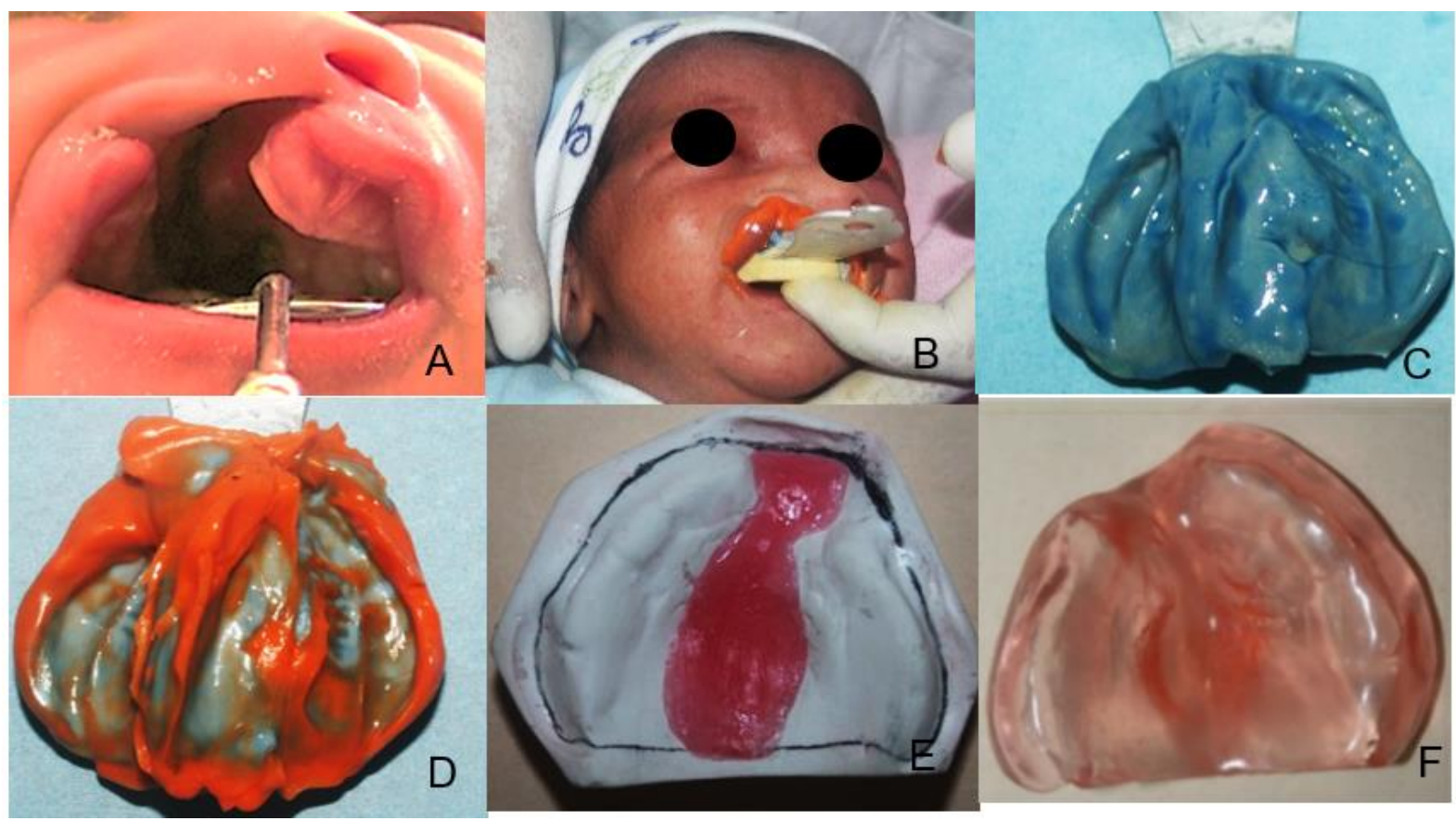

Figure 1: Realization Steps of Oral Molding Appliance.

(A) Pretreatment view (age: 2 days). (B, C, D) Impression of unilateral cleft defect. (E) Undercut block-outstand development of arch form in baseplate wax. (F) Oral Molding Appliance.

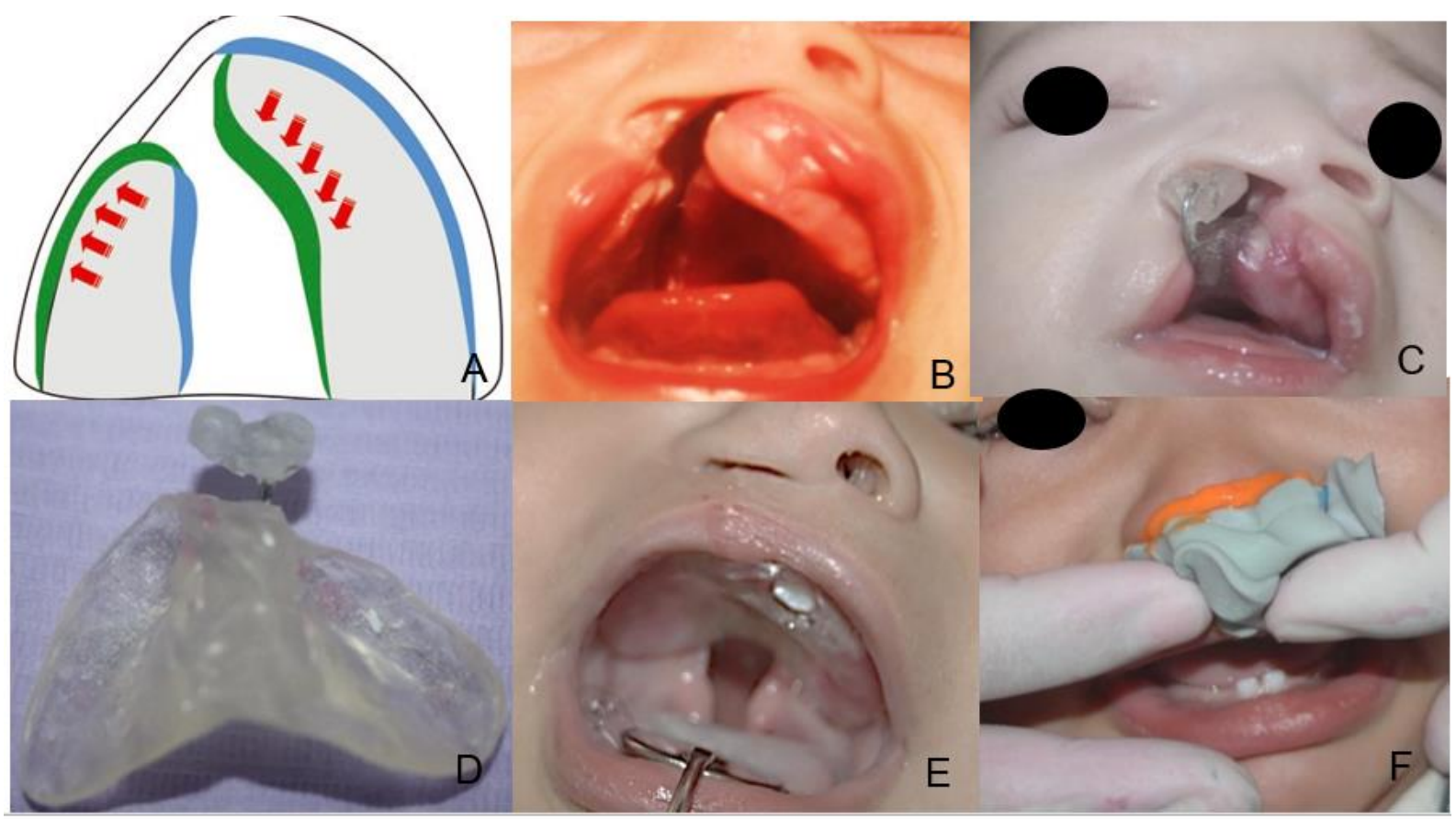

Figure 2: Realization Steps of NAM Device

(A) Schematic diagram of molding appliance: blue areas indicate soft liner application, and green areas indicate areas of relief. Red arrows indicate direction of force. (B) Close approximation of alveolar segments. (C) Nasal Stent: 19 gauge SS wire covered with acrylic and soft liner. (D) Nasal stent positioned in nostril aperture to support the nasal dome and pushing outward. (E) Cleft nasal deformity after nasoalveolar molding just before surgery. (F) Cleft nasal deformity after nasoalveolar molding just before surgery. 


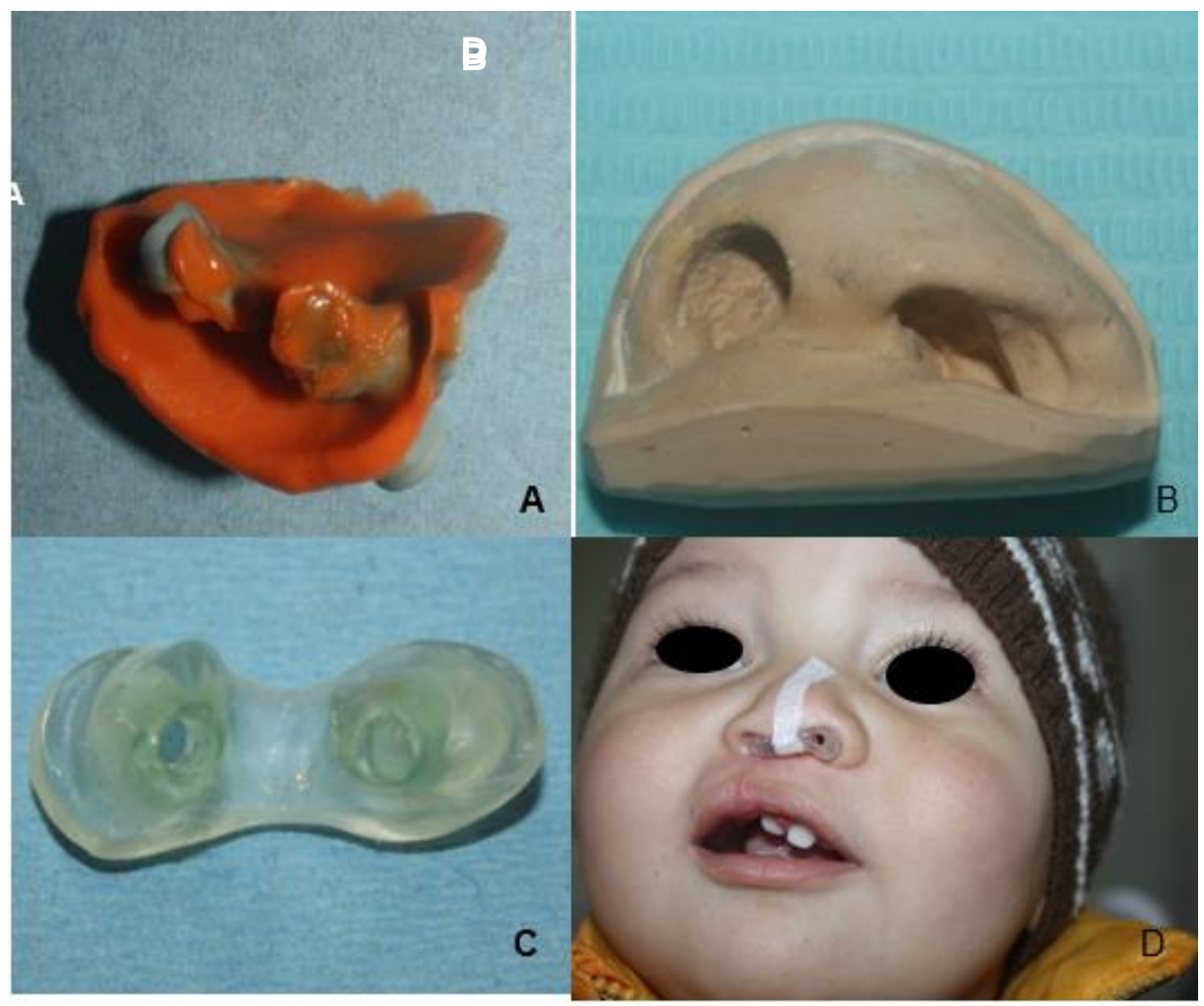

Figure 3: Realization Steps of Nostril Retainers

(A, B) Nostril Impression; (C) Nostril plaster model (D) Nostril retainers manufactured from orthodontic acrylic resin. (E) Post orthopedic treatment view (age: 18 months)

\section{DISCUSSION}

The effectiveness of preoperative orthopedic treatment for infants with CLP has long been debated and controversial. The points of controversy are diverse, not only related to the effectiveness of this treatment, but also, the time of treatment and the types of forces used.

The study of PUNGA and col ${ }^{i}$ consists of a comparison between a study group benefiting from NAM and a control group left without NAM. The two groups are each composed of ten newborns of less than two months of age, reviewed every 2-3 weeks until six months of cheiloplasty to record some measurements, for each visit, using calipers. This study concluded that there is a statistically significant difference between the study group and the control group, especially with respect to columellar length, as well as projection of the tip of the nose. Their result was in agreement with the studies of MAULL and al. ${ }^{\mathrm{ii}}$, CUTTING and col. ${ }^{\mathrm{iii}}$ and GRAYSON and $\mathrm{col}^{\mathrm{iv}}$.

GRAYSON and col. $^{4}$ described the complete data footprints and the design, manufacture and complications of treatment with NAM. They stated that the goal of orthopedic treatment is to reduce the severity of the original deformities and thus allow the surgeon to perform better repair of the alveolar bone, lips and nose. According to their work, the use of NAM also avoids columellar surgical reconstruction and scar tissue after the surgical procedure. Similarly, KIRBSCHUS and al. ${ }^{\mathrm{v}}$ reported the use of a device consisting of an acrylic button (attached to the palatal plate with a wire), which by activating it allows to lift the nasal dome and bring the edges of the slit the one to the other.
ROMERO and al. vi have used an orthopedic approach represented by NAM witch it is a device-implanted intrabone at the pre maxillary level. This device has two hooks located in front of and outside the dental crest, allowing the fixation of the elastic bands, which themselves have an orthopedic action. KIRBSCHUS and al. ${ }^{5}$ reported the use of a device consisting of an acrylic button (attached to the maxillary plate with a wire) which by activating it allows lifting the nasal dome and bringing the edges of the slit the one to the other. SINGH and col. ${ }^{\text {vii }}$ evaluated 3D facial morphology in patients surgically corrected for FLP after NAM. The study showed that nasal and palatal symmetry has improved before labial surgical repair. Nasal valve modeling indicated, postoperatively, to maintain the result obtained. MATSUO et al. ${ }^{\text {viii }}$ stated that nasal cartilage is flexible and plastic at birth, and congenital malformations can therefore easily corrected by NAM. However, as the child grows up, the flexibility of the nasal cartilage decreases. EZZAT and al. ${ }^{\text {ix }}$ evaluated the NAM results in the treatment of patients with CLP, the improvement of the height of the nasal cleft correlated with the moment when the device has applied. LIOU and al. ${ }^{\mathrm{x}}$ concluded that NAM and primary cheiloplasty both allow the lengthening of the columella in the bilateral clefts. SPENGLER and al. ${ }^{x i}$ showed that NAM has significant advantages in the treatment of CLP. PFEIFFER and al. ${ }^{\mathrm{xii}}$ introduced notion of gingivoperiostioplasty in the management of clefts. They concluded from NAM of CLP associated with early gingivoperiosclerosis, leading to substantial savings, compared to secondary alveolar 
bone graft treatment. Contrary to the results of previous studies on the long-term effect of NAM on the width of the Cleft, in this case the PUNGA and col. ${ }^{3}$, which joins the results of our work, YAMADA et al. xiii concluded that preoperative orthopedics reduces the width of the cleft and thus facilitates reconstruction surgery. According to TALMANT ${ }^{\mathrm{xiv}}$, the therapeutic chronology of CLP does not go through an NAM. At the age of 6 months, labial and velar closure has performed. Then, we maintain a modeling by nasal valve for 3 to 4 months. At 18 months, the bony palate has anatomically closed in two planes, with no area left for secondary healing. Between 4 and 5 years of age, gingivoperiostioplasty has performed, possibly with an iliac bone graft. From the age of six, orthodontic treatment begins. RINGDAHL ${ }^{\mathrm{xv}}$ conducted a retrospective study on the long-term effects of NAM and facial growth in patients with CLP. Their sample has composed of 28 patients, 16 of whom benefited from NAM and 12 treated surgically without NAM. The authors used photographs, study models and lateral teleradiography to compare the two groups before and after the surgery. This study concluded that there were no significant effects of orthopedic treatment on nasolabial shape and symmetry in post cheiloplasty.

\section{CONCLUSION}

Although there are still a few schools that neglect the effect of NAM in the management of CLP, the majority of authors agree that this therapy nevertheless provides

\section{REFERENCES}

[1] Punga Rohit, SM Sharma. J. Presurgical Orthopedic Nasoalveolar Molding in Cleft Lip and Palate Infants: A Comparative Evaluation of Done Cases with and Without Nasal Maxillofacial Stents. Oral Surg. (July-Sept 2013) 12 (3): 273-288.

[2] Maull DJ, Grayson BH, CB Cutting, Brecht LL, Bookstein FL, Khorrambadi D et al. Long term effects of nasoalveolar molding on three-dimensional nasal shape in unilateral clefts. Cleft Palate Craniofacial J (1999) 36: 391-397.

[3] Cutting C, Grayson B, Brecht L, Santiago P, Wood R, Kwon S. Presurgical columellar elongation and primary retrograde nasal reconstruction in one-stage bilateral cleft lip and nose repair. Plastic Reconstr Surg. (1998) 101: 630-639.

[4] Grayson BH, Shetye PR, Cutting CB. Presurgical nasoalveolar molding treatment in cleft lip \& palate patients. Cleft J (2005) 1: 4-7.

[5] Kirbschus A, Gesch D, Heinrich A, Gedrange $T$ Presurgical nasoalveolar molding in patients with unilateral clefts of lip, alveolus and palate. Case study and review of literature. J CranioMaxillofac Surg (2006) 34: $45-48$

[6] Romero M, Latham R, Romance A, Salvan R Treatment of an infant with a rare cleft resolved with use of an orthopedic appliance. Cleft Palate Craniofac J (2003) 40: 642-644.

[7] Singh GD, Bercowski DL, and Santiago PE Threedimensional nasal changes following nasoalveolar molding in patients with unilateral cleft lip and palate: geometric morphometrics. Cleft Palate Craniofac J. (2005) 42: 403-409.

[8] Matsuo K, Hirose T, Otagiri T, Norose N Repair of cleft lip with non-surgical correction of nasal deformity in the very significant results, compared to surgical treatment without orthopedic prerequisite. Indeed, NAM concretely influences the shape and symmetry of the nose and therefore a harmonious facial growth in the long term. In contrast, no significant effect of orthopedic treatment on maxillary arches and subsequent development of dentition and occlusion has demonstrated. In addition, there is no study endorsing the effect of NAM on oral functions

\section{AUTHORS' CONTRIBUTIONS}

The participation of each author corresponds to the criteria of authorship and contributorship emphasized in the Recommendations for the Conduct, Reporting, Editing, and Publication of Scholarly work in Medical Journals of the International Committee of Medical Journal Editors. Indeed, all the authors have actively participated in the redaction, the revision of the manuscript, and provided approval for this final revised version.

\section{ACKNOWLEDGMENTS}

None.

\section{COMPETING INTERESTS}

The authors declare no competing interests with this case.

\section{FUNDING SOURCES}

None.

early neonatal period. Plastic Reconstr Surg (1989) 83; 25-31.

[9] Ezzat CF, Chavarria C, Teichgraeber JF. Presurgical nasoalveolar molding therapy for the treatment of unilateral cleft lip and palate: a preliminary study. Cleft Palate Craniofac J (2007) 44: 8-12.

[10] Liou EJW, Subramanian M, Chen PKT Progressive changes in columella length and nasal growth after nasoalveolar molding in bilateral patients: a 3-year follow-up study. Plastic Reconstr. Surg (2007) 119: 642648

[11] Spengler AL, Chavarria C, Teichgraeber JF, Gateno J, Xia JJ. Presurgical nasoalveolar molding therapy for the treatment of bilateral cleft lip and palate: a preliminary study. Cleft Palate Craniofacial J. (2006) 43: 321-328.

[12] Pfeifer TM, Grayson BH, CB Cutting. Nasoalveolar molding and gingivoperiosteoplasty versus alveolar bone graft: an outcome analysis of costs in the treatment of unilateral cleft alveolus. Cleft Palate Craniofac J. (2002) 39: 26-29.

[13] Yamada T, Mori Y, Mishima K, Sugahara T Nasolabial and alveolar morphology following presurgical orthopedic treatment in complete unilateral clefts of lip, alveolus and palate.J Cranio-Maxillofac Surg (2003) 31 : 343-347.

[14] Talmant JC. Cleft rhinoplasty, from primary to secondary surgery. Ann Chir Plast Esthet. 2014 Dec; 59 (6): 555-84. Doi: 10.1016 / j.anplas.2014.08.004.

[15] Ringdahl Lindsay. Long-term effect of nasoalveolar molding on midface growth and nasolabial aesthetics in complete unilateral cleft lip and palate patients. Master's thesis 12-2011. Nova Southeastern University. Retrieved from NSUWorks, College of Dental Medicine. http://nsuworks.nova.edu/hpd_cdm_stuetd (visited on 19/11/2016 at 17:45). 